\title{
FREE CONVECTIVE FLOW OF A STRATIFIED FLUID THROUGH A POROUS MEDIUM BOUNDED BY A VERTICAL PLANE
}

\author{
H.K. MONDAL and T.K. CHAUDHURY \\ Department of Mathematics \\ University of Burdwan \\ Burdwan \\ West Bengal, India \\ (Received April 16, 1991 and in revised form December 30, 1992)
}

\begin{abstract}
Steady two-dimensional free convection flow of a thermally stratified viscous fluid through a highly porous medium bounded by a vertical plane surface of varying temperature, is considered. Analytical expressions for the velocity, temperature and the rate of heat transfer are obtained by perturbation method. Velocity distribution and rate of heat transfer for different values of parameters are shown in graphs. Velocity distribution is also obtained for certain values of the parameters by integrating the coupled differential equations by Runge-Kutta method and compared with the analytical solution. The chief concern of the paper is to study the effect of equilibrium temperature gradient on the velocity and the rate of heat transfer.
\end{abstract}

KEY WORDS AND PHRASES. Free convection, porous medium and stratified fluid. 1991 AMS SUBJECT CLASSIFICATION CODES. 76R10, 76V05.

\section{INTRODUCTION}

The investigation of problems involving free convection flow is invariably connected with the concept of stratification which means the stable variation of the density of the fluid in the gravitational field. The Boussinesq approximation is widely used in the case of little stratification in which it is customary to consider the small deviation of the density from some constant value. However, the introduction of the analogous deviation from the hydrostatic value is more natural and Turchak and Shidlovskii [1] have derived from this approach the system of equations of motion for a stratified fluid in Boussinesq approximation in the presence of viscosity, thermal conductivity and diffusion. This system of equations of Turchak and Shidlovskii thus introduces explicitly the effect of equilibrium stratification through the equilibrium density gradient arising in general, from equilibrium salinity and temperature gradients. For a thermally stratified fluid equilibrium temperature gradient only appear.

Raptis et al. [2] studied the free convection flow of an incompressible viscous fluid through a porous medium bounded by a vertical porous plate with constant temperature considering viscous dissipation only. Rudriah and Nagaraj [3] investigated for free convection flow of a viscous fluid in a porous medium bounded by two vertical plates kept at constant temperature and considered both viscous and Darcy dissipations. However, the above references do not consider the fluid to be stratified in the sense that the equilibrium temperature of the fluid is taken there to be uniform.

Here we investigate the two-dimensional free convection flow of a thermally stratified viscous fluid through a porous medium bounded by a heated vertical plate taking into account both the viscous and Darcy dissipations, the temperature of the plate varying linearly along the vertical direction. The main object here is to find the effect of equilibrium temperature gradient of the fluid on the flow. 
Assuming the porous medium as an assemblage of small identical spherical particles fixed in space the equation for incompressible fluid can be taken in the form [4],

$$
\rho^{\prime}\left(\overrightarrow{q^{\prime}} \cdot \nabla\right) \overrightarrow{q^{\prime}}=-\nabla p-\frac{\mu}{k^{\prime}} \vec{q}^{\prime}+\mu \nabla \frac{2}{q^{\prime}}-\rho^{\prime} \vec{g}
$$

where $\vec{q}^{\prime}$ is the velocity, $\rho^{\prime}$ the density, $p$ the pressure, $\mu$ the viscosity of the fluid, $k^{\prime}$ the permeability of the medium and $\vec{g}$ the acceleration due to gravity.

\section{FORMULATION OF THE PROBLEM}

We take a Cartesian coordinate system with the $y^{\prime}$-axis vertically upward along the plate and the $x^{\prime}$-axis normal to it. Now the temperature, density and pressure of the fluid can be written as (Ref. [1])

$$
T^{\prime}=T_{c}+\theta^{\prime}, \quad \rho^{\prime}=\rho_{c}-\alpha \rho_{0} \theta^{\prime} \text { and } p=p_{c}+\bar{p},
$$

where $T_{e}, \rho_{e}, p_{e}$ the equilibrium temperature, density, pressure respectively of the fluid, are given by $T_{e}=T_{0}+A_{T}^{\prime} y^{\prime}, \rho_{e}=\rho_{0}\left(1-\alpha T_{e}\right)$ and $\frac{d \rho_{e}}{d y^{\prime}}=-\rho_{e} g$; and $\theta^{\prime}, \bar{p}$ are the deviation of temperature and pressure, the constant $A_{T}{ }^{\prime}(>0)$ is the equilibrium temperature gradient of the fluid, the constants $\rho_{0}$ and $T_{0}$ are the reference density and temperature respectively and $\alpha$ is the coefficient of volume expansion. We assume the surface temperature of the plate in the form $T_{w}{ }^{\prime}=T_{W}+T_{e}$, where $T_{w}(>0)$ is a constant.

Here the motion is essentially due to the temperature gradient in the plate. The plate being assumed infinite along the $y^{\prime}$-axis, the field variables $\theta^{\prime}$ and $\vec{q}^{\prime}$ are taken to be independent of $y^{\prime}$. Then using the equation of continuity and applying Boussinesq approximation the equation of motion for the problem (cf. (1.1)) becomes

$$
v \frac{d^{2} v^{\prime}}{d x^{\prime 2}}-\frac{v}{k^{\prime}} v^{\prime}+\alpha g \theta^{\prime}=0
$$

and the equation of energy becomes

$$
\kappa \frac{d^{2} \theta^{\prime}}{d x^{\prime 2}}+\rho_{0} v\left(\frac{d v^{\prime}}{d x^{\prime}}\right)^{2}+\frac{v \rho_{0}}{k^{\prime}} v^{\prime 2}-C_{p} \rho_{0} A_{T}^{\prime} v^{\prime}=0
$$

The boundary conditions are

$$
\left.\begin{array}{cccc}
v^{\prime}=0, & \theta^{\prime}=T_{w} & \text { at } & x^{\prime}=0 \\
v^{\prime} \rightarrow 0, & \theta^{\prime} \rightarrow 0 & \text { as } & x^{\prime} \rightarrow \infty
\end{array}\right]
$$

where $v^{\prime}$ is the velocity component along the $y^{\prime}$-axis, $\mathrm{K}$ is the thermal conductivity, $C_{p}$ is the specific heat at constant pressure, $v-\mu / \rho_{0}$ and the second and the third term in the energy equation (2.2) represent the viscous and Darcy dissipation respectively.

Introducing the non-dimensional variables $v=v^{\prime} / \beta, x=x^{\prime} \beta / v, \theta=\theta^{\prime} / T_{W}$ where $\beta=\left(\alpha g v T_{W}\right)^{1 / \beta}$ has dimension of velocity, the above equations with the boundary conditions take the form

$$
\begin{gathered}
\frac{d^{2} v}{d x^{2}}-\sigma^{2} v+\theta=0 \\
\frac{d^{2} \theta}{d x^{2}}+N\left(\frac{d v}{d x}\right)^{2}+N \sigma^{2} v^{2}-P A_{T} v=0
\end{gathered}
$$

with

$$
v=0, \theta=1 \text { at } x=0
$$

and

$$
v \rightarrow 0, \quad \theta \rightarrow 0 \text { as } x \rightarrow \infty
$$

where $\sigma=v / \sqrt{k^{\prime}} \beta$ (Darcy resistance parameter), $N=\rho_{0} v \beta^{2} / \kappa T_{W}$ (buoyancy force parameter), $P=\rho_{0} C_{p} v / \kappa$ (Prandtl number) and $A_{T}=v A_{T}^{\prime} / T_{\mathrm{W}} \beta$ (equilibrium temperature gradient parameter). 


\section{SOLUTION BY PERTURBATION METHOD}

The coupled equations (2.4) and (2.5) are non-linear due to dissipation terms. However, in many practical problems $N$ is small and therefore, with $N$ as a perturbation parameter we can employ the perturbation technique to solve the above equations.

We write

$$
\left.\begin{array}{l}
v(x)=v_{0}(x)+N v_{1}(x)+0\left(N^{2}\right) \\
\theta(x)=\theta_{0}(x)+N \theta_{1}(x)+0\left(N^{2}\right)
\end{array}\right]
$$

where $v_{0}, \theta_{0}$ are the solutions corresponding to $N=0$ i.e., when the dissipations are neglected.

The sets of equations of different orders can then be obtained as

$$
\text { zeroth order: } \left.\begin{array}{rl}
v_{0}{ }^{\prime \prime}-\sigma^{2} v_{0}+\theta_{0} & =0 \\
\theta_{0}{ }^{\prime \prime}-P A_{T} v_{0} & =0
\end{array}\right]
$$

together with the corresponding boundary conditions

$$
\begin{aligned}
& v=0, \quad v_{1}=0, \quad \theta_{0}=1, \quad \theta_{1}=0 \quad \text { at } \quad x=0 \\
& v_{0} \rightarrow 0, \quad v_{1} \rightarrow 0, \quad \theta_{0} \rightarrow 0, \quad \theta_{1} \rightarrow 0 \quad \text { as } \quad x \rightarrow \infty
\end{aligned}
$$

where primes denote differentiation with respect to $x$.

Solving the equations (3.2) and (3.3) with the help of (3.4) we get $v_{0}, \theta_{0}$ and $v_{1}, \theta_{1}$ respectively and from (3.1) we finally get the expressions of velocity and temperature in the form

$$
\begin{gathered}
v=M\left(e^{-\beta_{1} x}-e^{-\alpha_{1} x}\right)+N\left[A_{1} e^{-\alpha_{1} x}+A_{2} e^{-\beta_{1} x}+M^{2}\left\{\frac{\left(\alpha_{1}^{2}+\sigma^{2}\right) e^{-2 \alpha_{1} x}}{3 \alpha_{1}^{2}\left(4 \alpha_{1}^{2}-\beta_{1}^{2}\right)}\right.\right. \\
\left.\left.+\frac{\left(\beta_{1}^{2}+\sigma^{2}\right)}{3 \beta_{1}^{2}\left(4 \beta_{1}^{2}-\alpha_{1}^{2}\right)} e^{-2 \beta_{1} x}-\frac{2\left(\alpha_{1} \beta_{1}+\sigma^{2}\right)}{\alpha_{1} \beta_{1}\left(\beta_{1}+2 \alpha_{1}\right)\left(\alpha_{1}+2 \beta_{1}\right)} e^{-\left(\alpha_{1}+\beta_{1}\right) x}\right\}\right] \\
\theta=\left(M+A_{2} N\right) \alpha_{1}^{2} e^{-\beta_{1} x}+\left(A_{1} N-M\right) \beta_{1}^{2} e^{-\alpha_{1} x}+N M^{2}\left\{\frac{\left(\alpha_{1}^{2}+\sigma^{2}\right)\left(\sigma^{2}-4 \alpha_{1}^{2}\right)}{3 \alpha_{1}^{2}\left(4 \alpha_{1}^{2}-\beta_{1}^{2}\right)} e^{-2 \alpha_{1} x}\right. \\
\left.+\frac{\left(\beta_{1}^{2}+\sigma^{2}\right)\left(\sigma^{2}-4 \beta_{1}^{2}\right)}{3 \beta_{1}^{2}\left(4 \beta_{1}^{2}-\alpha_{1}^{2}\right)} e^{-2 \beta_{1} x}+\frac{4\left(\alpha_{1} \beta_{1}+\sigma^{2}\right)}{\left(\beta_{1}+2 \alpha_{1}\right)\left(\alpha_{1}+2 \beta_{1}\right)} e^{-\left(\alpha_{1}+\beta_{1}\right) x}\right\}
\end{gathered}
$$

where

$$
\begin{gathered}
\alpha_{1}=\left(\frac{\sigma^{2}+\sqrt{\lambda}}{2}\right)^{1 / 2} \beta_{1}=\left(\frac{\sigma^{2}-\sqrt{\lambda}}{2}\right)^{1 / 2} \text { for } \lambda>0 \\
A_{1}=-\frac{1}{\lambda^{3 / 2}}\left[\frac{\alpha_{1}^{2}+\sigma^{2}}{3 \alpha_{1}^{2}}+\frac{\beta_{1}^{2}+\sigma^{2}}{4 \beta_{1}^{2}-\alpha_{1}^{2}}-\frac{2\left(\alpha_{1} \beta_{1}+\sigma^{2}\right)}{\beta_{1}\left(\beta_{1}+2 \alpha_{1}\right)}\right], A_{2}=\frac{1}{\lambda^{3 / 2}}\left[\frac{\alpha_{1}^{2}+\sigma^{2}}{4 \alpha_{1}^{2}-\beta_{1}^{2}}+\frac{\beta_{1}^{2}+\sigma^{2}}{3 \beta_{1}^{2}}-\frac{2\left(\alpha_{1} \beta_{1}+\sigma^{2}\right)}{\alpha_{1}\left(\alpha_{1}+2 \beta_{1}\right)}\right] \\
M=1 /\left(\alpha_{1}^{2}-\beta_{1}^{2}\right) \text { and } \lambda=\sigma^{4}-4 P A_{T} .
\end{gathered}
$$

Again for $\lambda<0, \alpha_{1}$ and $\beta_{1}$ will become complex and the real part of (3.5) and (3.6) will be the solutions in this case. We can also obtain a solution in the case $\lambda=0$ i.e., $A_{T}=\sigma^{4} / 4 P$.

For very high permeability of the medium, the parameter $K\left(=\frac{1}{\sigma^{2}}\right)$ will be very large and the solution of the corresponding problem for the free flow of the fluid is obtained by making $\sigma \rightarrow 0$ in our solution.

We can calculate the shear stress $\tau=\left(\frac{d v}{d x}\right)_{x=0}$ at the plate from (3.5) and the rate of heat transfer between the fluid and the plate in terms of the Nusselt number $N u$, as $N u=-\left(\frac{d \theta}{d x}\right)_{x=0}$ from (3.6). 


\section{SOLUTION BY NUMERICAL METHOD}

The coupled second order ordinary differential equations (2.4) and (2.5) with boundary conditions (2.6) and (2.7) are also solved numerically by fourth order Runge-Kutta method. For this we put the equations (2.4), (2.5) in the form

$$
\text { and } \begin{aligned}
\frac{d v}{d x} & =Y_{1}, \frac{d \theta}{d x}=Y_{2} \\
\frac{d Y_{1}}{d x} & =\sigma^{2} v-\theta \\
\frac{d^{2} Y_{2}}{d x} & =P A_{T} v-\mathrm{N}_{1}^{2}-N \sigma^{2} v^{2}
\end{aligned}
$$

together with the initial conditions (2.6) and

$$
Y_{1}=\frac{d v}{d x}=\tau, \quad Y_{2}=\frac{d \theta}{d x}=-N u \text { at } x=0
$$

The infinity conditions in (2.7) namely $(v \rightarrow 0, \theta \rightarrow 0$ as $x \rightarrow \infty)$ will be replaced by the condition $v, \theta<10^{-8}$ at $x \geq x_{n}$ where $x_{n}$ is a suitable value depending on the parameters. To find the initial conditions (4.2) for specified $P, A_{T}, \sigma$ and $N$, we apply an improvement method with the values of $\frac{d v}{d x}$ and $\frac{d \theta}{d x}$ at $x=0$, calculated from the zeroth order solution (i.e., when $N=0$ ) as the starting values. Let these starting values be $\alpha_{0}$ and $\beta_{0}$. Then the appropriate improvement of these values are carried out by an iteration scheme (Krishnamurthy and Sen [5-7]) until the infinity condition state above is satisfied.

The numerical solutions for $v$ and $\theta$ are computed for a fixed $P=0.71$ with different $A_{T}(0.05,0.1$, $0.3,0.5$ and 1.0$)$, different $\sigma(1$ and 2$)$ and different $N(0.01,0.1,0.2$ and 0.3$)$.

\section{DISCUSSION}

The coupled non-linear equations (2.4) and (2.5) for velocity and temperature are solved by perturbation method with the buoyancy force $N$ as perturbation parameter. These equations are also solved by fourth order Runge-Kutta (RK) method for different $A_{T}, \sigma$ and $N$ with $P=0.71$. The buoyancy force parameter $N$ represents here the effects of free convection currents and we take $N>0\left(T_{W}>0\right)$ which corresponds physically to an externally cooled surface.

The velocity profiles obtained by the two methods for different $N$ (with fixed $A_{T}$ and $\sigma$ ) are shown in Figure 1 for comparison. We find for $A_{T}=0.3$ the maximum difference in the velocity obtained by the two methods are $0.62 \%$ for $N=0.1,3.2 \%$ for $N=0.2$ and $9.7 \%$ for $N=0.3$. Thus for $N \leq 0.1$ the two solutions may be taken to be practically identical. The value of the shear stress at the plate obtained by the two methods, agree up to a slightly higher value of $N$. For example, the change in the shear stress for $A_{T}=0.3$ are $0.27 \%$ for $N=0.1,1.31 \%$ for $N=0.2$ and $4.12 \%$ for $N=0.3$. Similar is the case for the rate of heat transfer.

It is also seen from computations that the agreement between the solutions by the two methods for a fixed $N(\leq 0.1)$ is not significantly affected for changes in $A_{T}$ and $\sigma$.

The velocity profiles for different $A_{T}$ and $\sigma(N=0.01, P=0.71)$ are shown in Figures 2-4. Figures 5 and 6 show the variation of shear stress $\tau$ and the rate of heat transfer (in terms of Nusselt number $\mathrm{Nu}$ ) respectively at the plate with $A_{T}$ for $\sigma=1$ and 2 .

We find from Figures 2, 3 and 4 that an increase in velocity and an increase in permeability $K\left(=\frac{1}{\sigma^{2}}\right)$ results in an increase in velocity.

From Figures 5 and 6 we see that the shear stress at the plate decreases and the rate of heat transfer at the plate increases with the increase in equilibrium temperature gradient. Also the shear stress and the rate of heat transfer at the plate increases as $\sigma$ decreases i.e., the permeability increases. 
We calculate separately the effects of viscous and Darcy dissipations on the velocity and also on the shear stress and the rate of heat transfer at the plate. It is found that for $N>0$ the effect of both the dissipations is to increase the velocity as well as the shear stress at the plate. The rate of heat transfer is however, decreased by these dissipations. All this is because of heat addition by dissipation. The effect of these dissipations decrease with an increase in $A_{T}$.

It is important to note, as calculations show, that the effect of viscous dissipation is always smaller than that of Darcy dissipation and for $A_{T} \leq \sigma^{4} / 4 P$ the effect of viscous dissipation is negligibly small compared to that of Darcy dissipation.

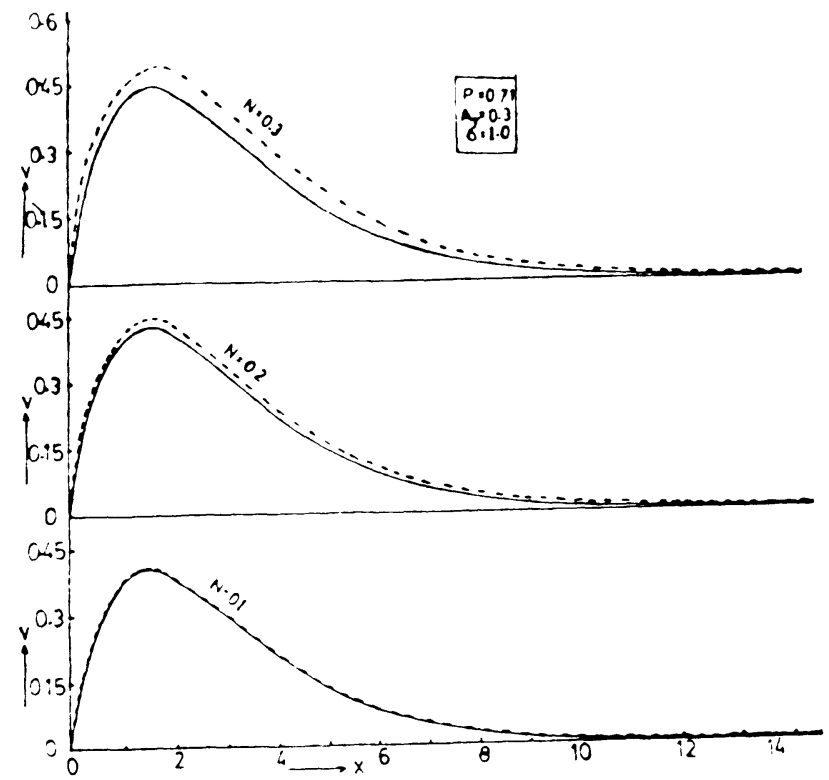

Figure 1. Solid line represents perturbation solution. Broken line represents numerical solution by RK method.

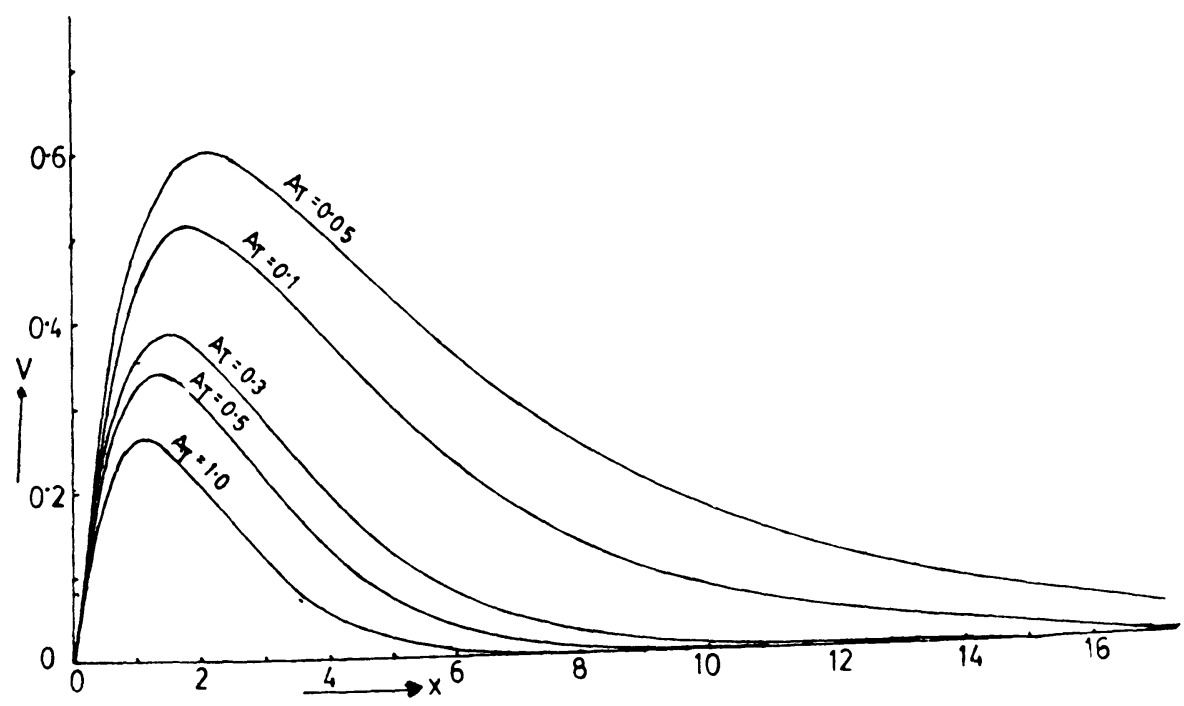

Figure 2. Velocity profiles for $P=0.71, N=0.01$ and $\sigma=1$. 


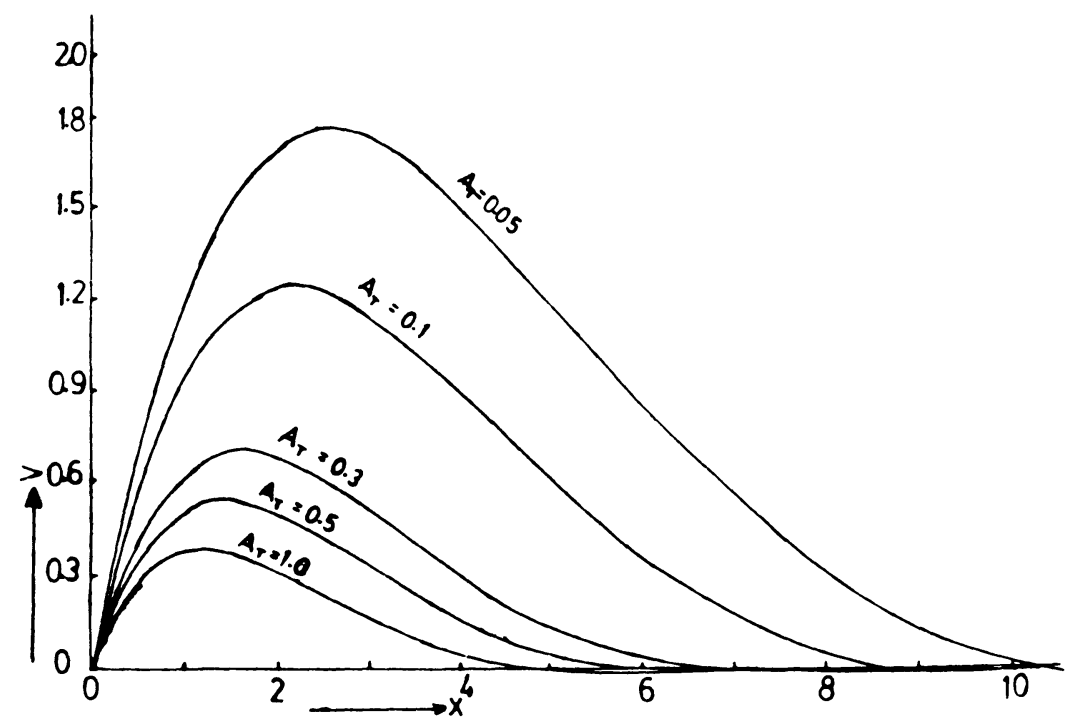

Figure 3. Velocity profiles for $P=0.71, N=0.01$ and $\sigma=2$.

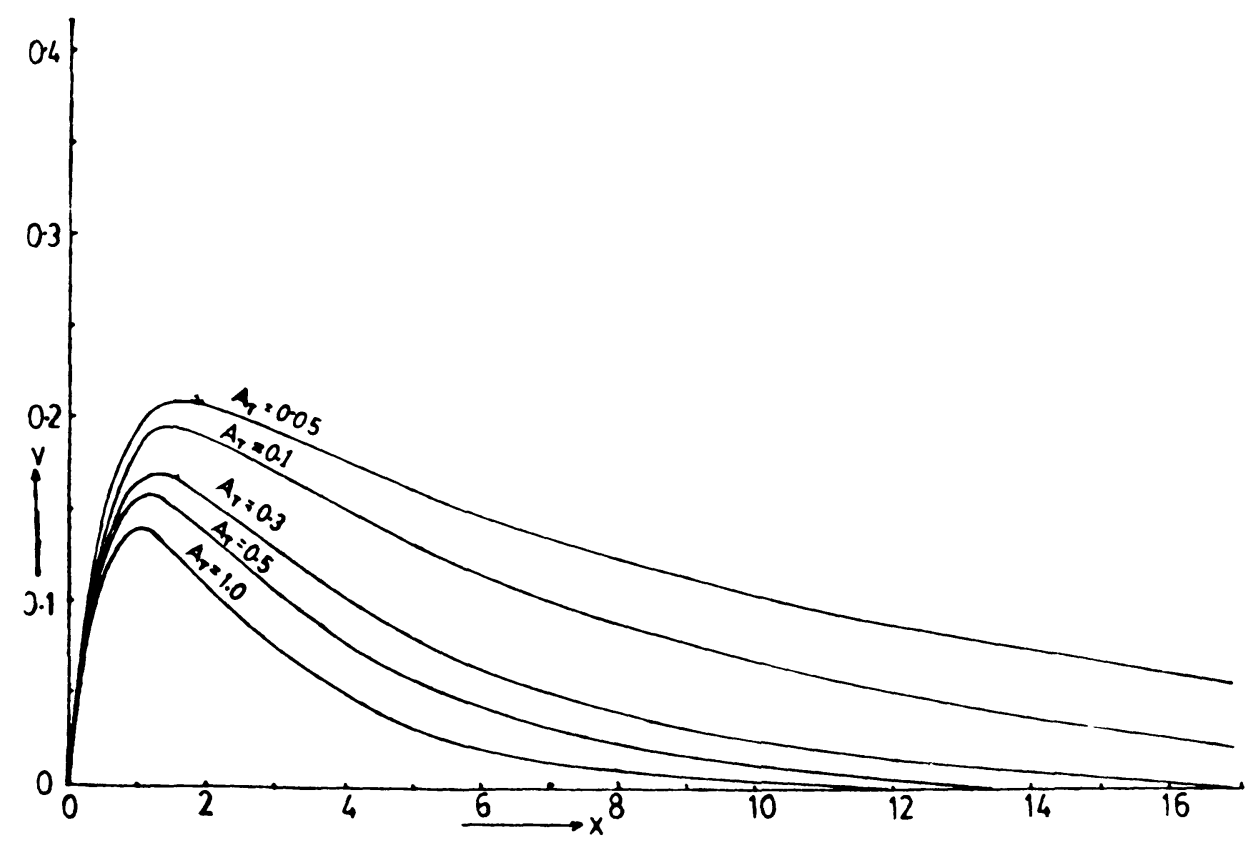

Figure 4. Velocity profiles in impervious medium for $P=0.71, N=0.01$. 


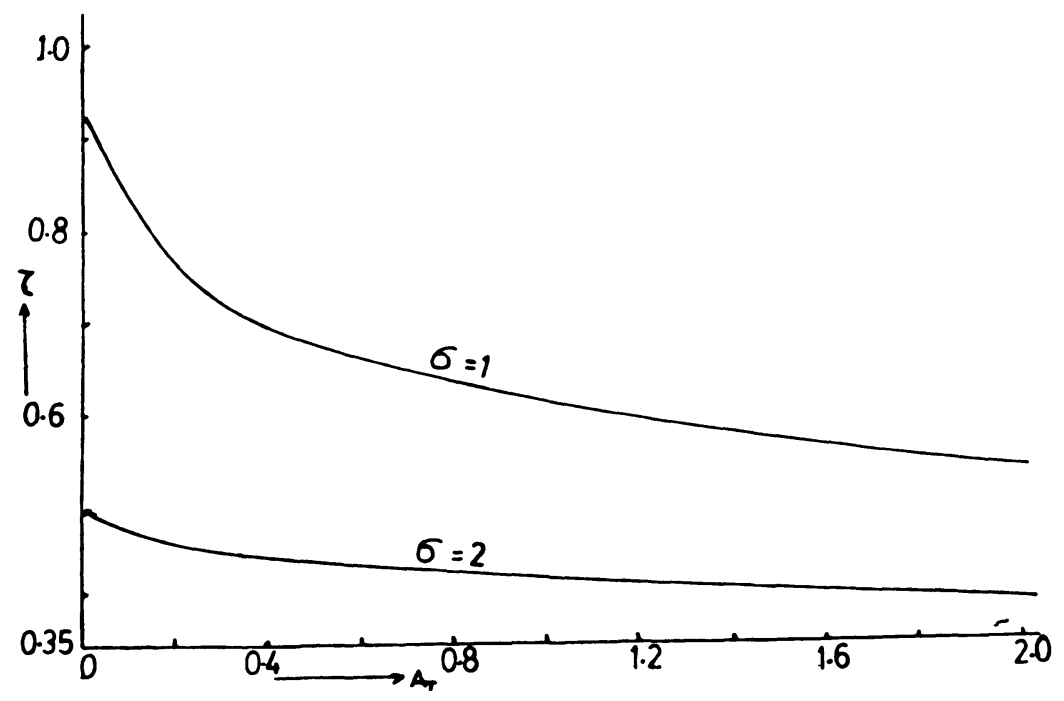

Figure 5. Shear stress at the plate with $A_{T}$ for $P=0.71, N=0.01$.

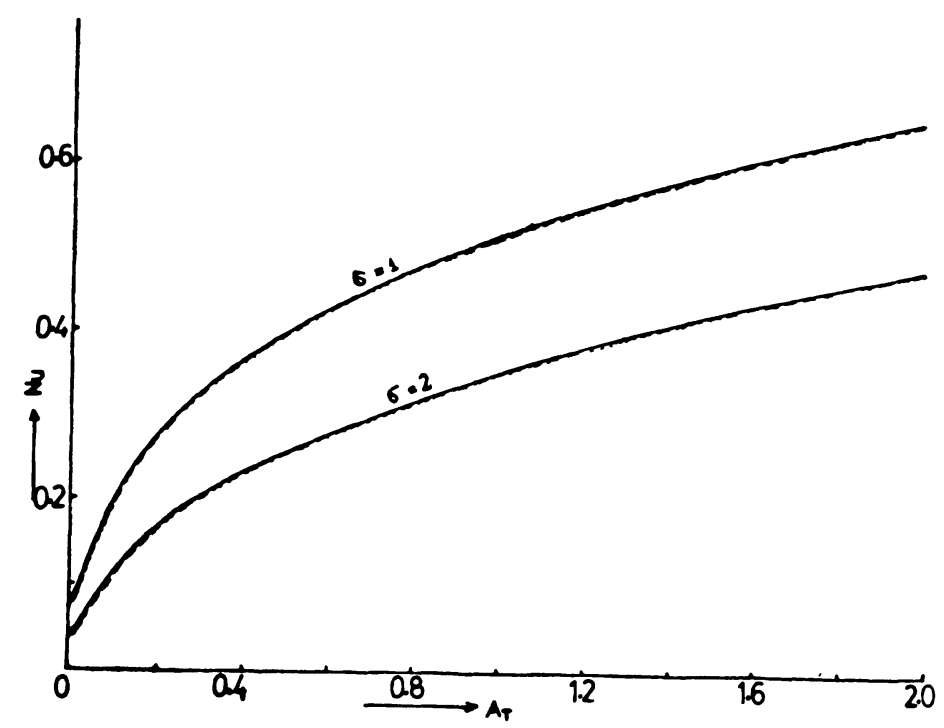

Figure 6. Variation in the rate of heat transfer in terms of Nusselt number with $A_{T}$ for $P=0.71, N=0.01$.

\section{REFERENCES}

1. TURCHAK, L. I. and SHIDLOVSKII, V. P. On the equations of motion of a stratified fluid, Dokl. Akad. Nauk. SSSR, 254 (1980), 317-321.

2. RAPTIS, A., PERDIKIS, C. and TZIVANIDIS, G. Free convection flow through a porous medium

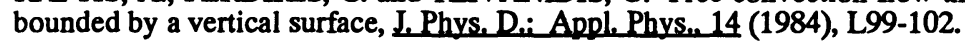

3. RUDRAIAH, N. and NAGARAJ, S. T. Natural convection through vertical porous stratum, Int. J Engng. Sci. 15, 9/10 (1977), 589-600.

4. RAPTIS, A. Unsteady free convective flow through a porous medium, Int. J. Engng. Sci. 21, 4 (1982), 345-348.

5. KRISHNAMURTHY, E. V. and SEN, S. K. Numerical algorithms, Affiliated East-West Press Pvt. LTD (1986), 459. 


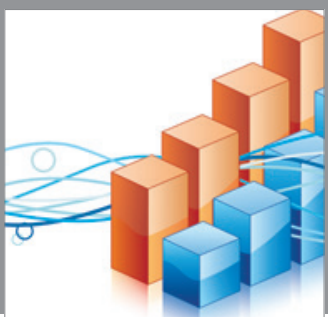

Advances in

Operations Research

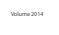

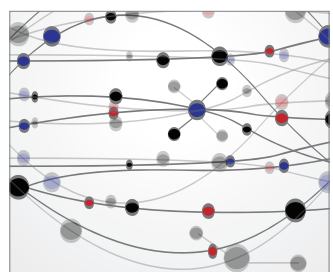

\section{The Scientific} World Journal
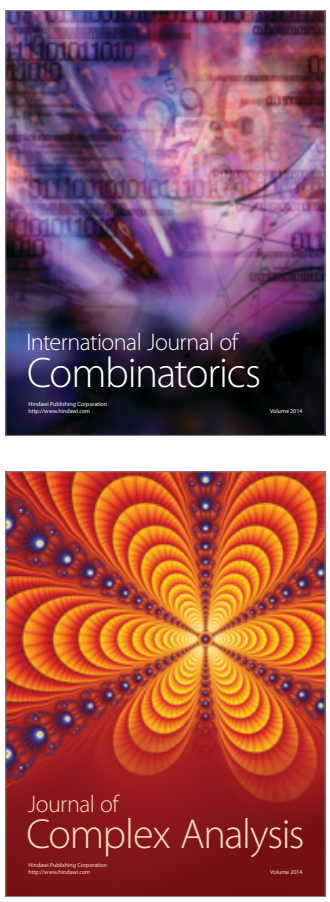

International Journal of

Mathematics and

Mathematical

Sciences
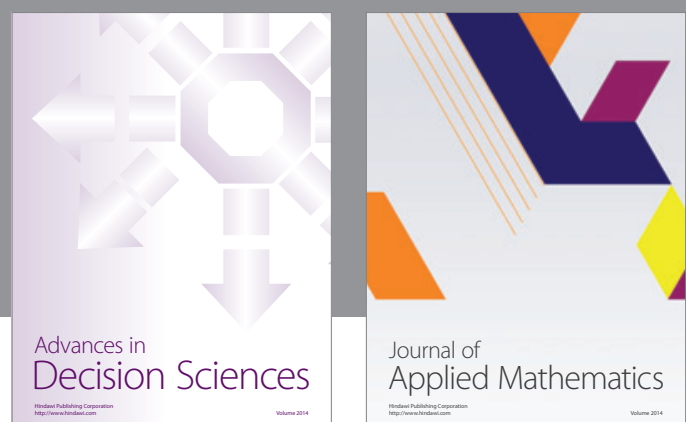

Journal of

Applied Mathematics
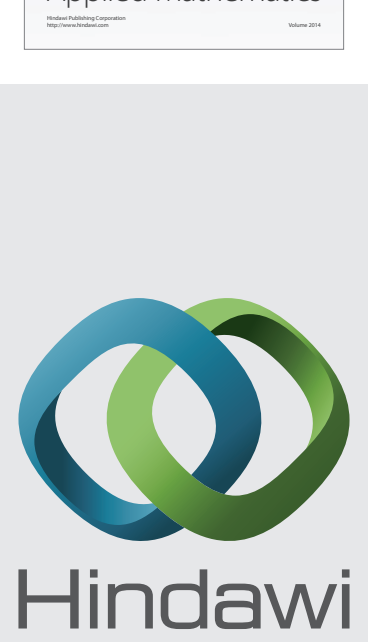

Submit your manuscripts at http://www.hindawi.com
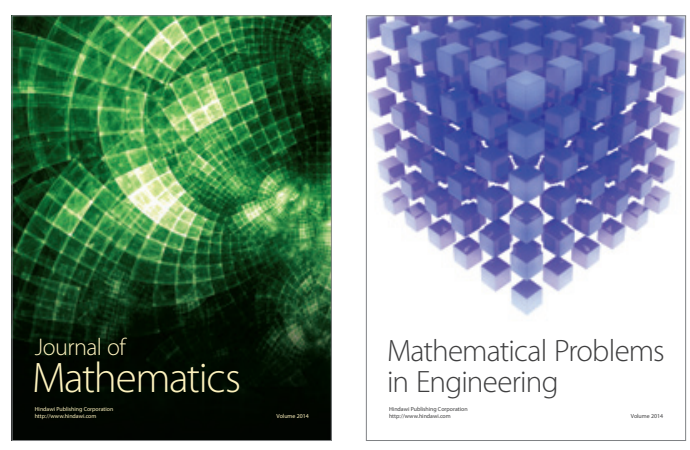

Mathematical Problems in Engineering
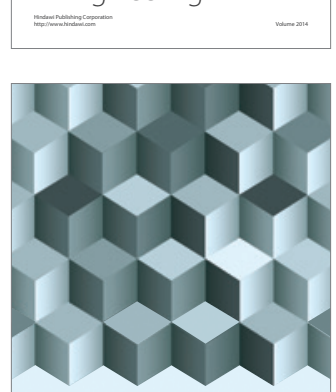

Journal of

Function Spaces
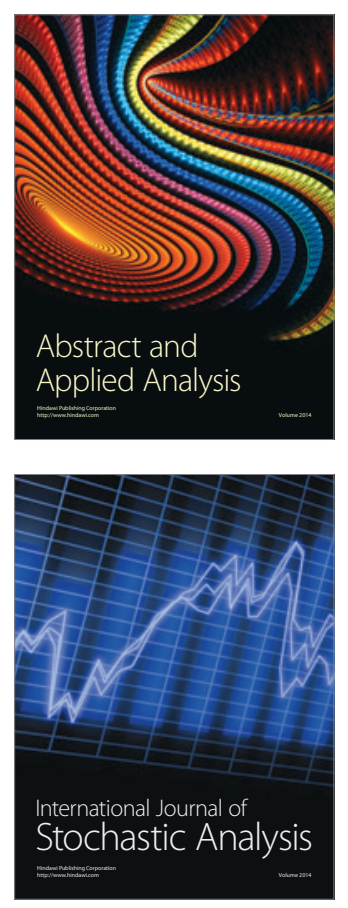

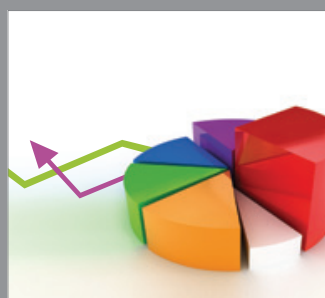

ournal of

Probability and Statistics

Promensencen
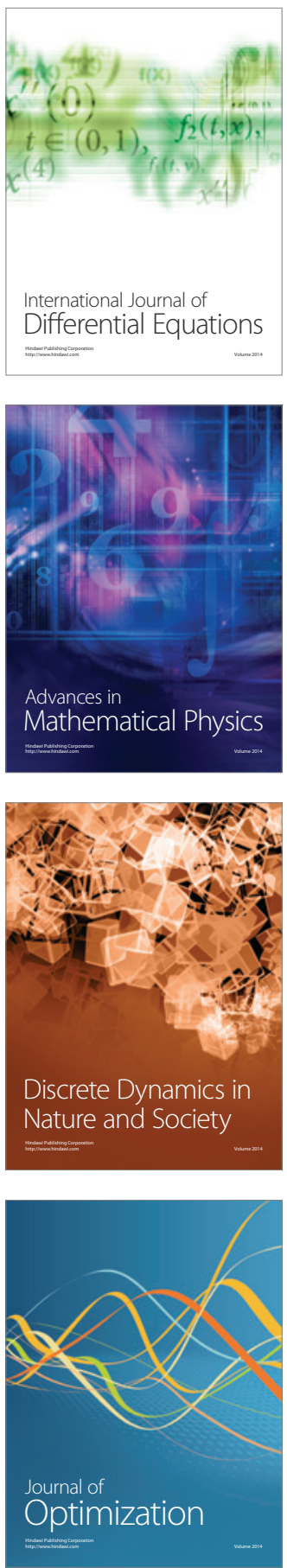